
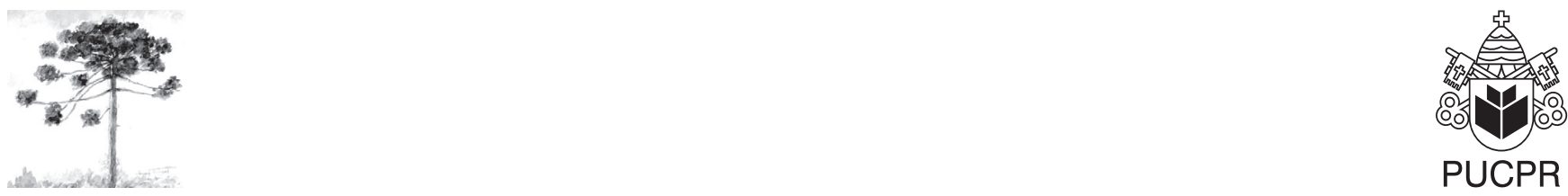

PUCPR

\title{
Ecologia alimentar de Piabina argentea Reinhardt, 1867 (Teleostei, Characidae) no Rio das Almas, São Paulo, Brasil
}

\author{
Feeding ecology of Piabina argentea Reinhardt, 1867 \\ (Teleostei, Characidae) in the Almas River, São Paulo, Brazil
}

Igor Kintopp $^{[a]}$, Vinícius Abilhoa ${ }^{[\mathrm{b}]}$

[a] Biólogo, Grupo de Pesquisas em Ictiofauna, Museu de História Natural Capão da Imbuia, Prefeitura de Curitiba, Curitiba, PR Brasil, e-mail: igor_kintopp@hotmail.com

[b] Biólogo, Doutor, Grupo de Pesquisas em Ictiofauna, Museu de História Natural Capão da Imbuia, Prefeitura de Curitiba, Curitiba, PR - Brasil, e-mail: vabilhoa@uol.com.br

\section{Resumo}

Neste estudo foram investigadas variações ontogenéticas e temporais na dieta de caracídeos Piabina argentea capturados no Rio das Almas, Alto Paranapanema, região sul de São Paulo. Amostras foram obtidas em junho de 2004, maio de 2006, novembro de 2006 e novembro de 2007. A dieta, avaliada por meio de uma matriz de similaridade com os dados de abundância dos itens alimentares, incluiu ácaros aquáticos, aranhas, microcrustáceos, decápodes, oligoquetos aquáticos, moluscos, insetos aquáticos e terrestres, estágios imaturos aquáticos de insetos, larvas de peixes, algas e restos de vegetais superiores. As maiores riquezas de itens alimentares foram observadas para as categorias de estágios imaturos aquáticos de insetos e insetos terrestres. A espécie apresentou hábito alimentar onívoro e sua dieta foi composta por material autóctone (principalmente estágios imaturos aquáticos de insetos) e alóctone (principalmente insetos terrestres e fragmentos de vegetais superiores). Muito embora a onivoria seja comum em caracídeos de pequeno porte que vivem em rios e riachos neotropicais, os resultados encontrados também indicam que os indivíduos maiores $(>5,6 \mathrm{~cm})$ apresentam preferência por itens vegetais, destacando a importância da mata ciliar para esses ecossistemas.

Palavras-chave: Alimentação. Caracídeo. Dieta. Onivoria. Ontogenia. 


\begin{abstract}
Ontogenetic and temporal variation in the diet of the characin Piabina argentea sampled in the Almas River, Upper Paranapanema River basin, southern São Paulo, were investigated. Samples were made in june 2004, may 2006, november 2006 and november 2007. The diet, evaluated by a similarity matrix with the abundance values of food items, included aquatic ticks, spiders, microcrustaceans, decapods, aquatic oligochaets, mollusks, aquatic insects, and terrestrial insects, aquatic immature insects (larvae and pupae), fish larvae, algae and plant fragments. Aquatic immature insects and terrestrial insects were the most representative feeding category. The characin $\mathrm{P}$. argentea presented an omnivorous feeding habit, and its diet was composed of autoch thonous (mainly aquatic immature insects) and allochthonous (terrestrial insects and plant fragments) material. Although the omnivore habit is considered common for small characins that inhabit neotropical streams and rivers, the results also indicate that the larger individuals $(>5.6 \mathrm{~cm})$ preferred plant fragments, highlighting the importance of ciliary forests for these ecosystems.
\end{abstract}

Keywords: Characin. Diet. Feeding. Omnivory. Ontogeny.

\section{Introduçáo}

O lambari Piabina argentea Reinhardt, 1867, é um caracídeo de pequeno porte (atinge cerca de 8 $\mathrm{cm}$ ) que apresenta ampla distribuição nas bacias hidrográficas do Rio Paraná, Rio São Francisco e diversas drenagens do leste do Brasil (1). Espécies de peixes de pequeno porte correspondem a pelo menos $50 \%$ do total de espécies de peixes de água doce descritas para a América do Sul, e mostram um grau elevado de endemismo geográfico $(2,3)$. Essas espécies são fortemente dependentes do material orgânico alóctone importado da vegetação marginal para sobreviver (4-6), estando fortemente ameaçadas por perturbações no ambiente, tais como: desmatamentos, degradação da qualidade da água e barramentos.

Os poucos estudos realizados com a espécie registraram sua maior ocorrência na região litorânea dos rios, e uma preferência alimentar por itens alóctones, demonstrando a importância da vegetação ciliar como fonte de nutrientes para a espécie (7). De fato, as matas ciliares são de suma importância para alimentação, abrigo e manutenção da diversidade desses ecossistemas $(8,9)$.

Muito embora os peixes tropicais apresentem considerável plasticidade trófica, ou seja, mudem sua dieta de acordo com as variações espaciais e temporais na disponibilidade dos itens alimentares (4), nos ambientes de produtividade primária relativamente baixa, onde os processos são predominantemente heterotróficos e de carreamento, a ictiofauna depende da importação da matéria alóctone para a sua subsistência (3).
Dentro desse contexto, o objetivo deste trabalho é descrever as variações ontogênicas e temporais na alimentação de exemplares de $P$. argentea coletados em um trecho do rio das Almas em São Paulo. A avaliação da forma de utilização dos recursos pelos organismos pode ser considerada como ferramenta importante para o entendimento das interações ecológicas entre as espécies, sendo essas informações fundamentais para projetos futuros de conservação de fauna aquática da Mata Atlântica.

\section{Materiais e métodos}

Os exemplares de $P$. argentea foram capturados em junho de 2004, maio de 2006, novembro de 2006 e novembro de 2007. As coletas foram realizadas em um trecho do Rio das Almas localizado entre as coordenadas 48W 20'55", 24S 09'25" e 48W 19'44", 24S 07'15", no município de Ribeirão Grande, São Paulo. Nesse trecho, o substrato do rio é formado principalmente por areia, cascalho e matéria vegetal (galhos, troncos de árvores e folhas), e as margens apresentam mata ciliar relativamente conservada (Figura 1).

Para a captura foram utilizadas redes de arrasto de malha de $5 \mathrm{~mm}$ e tarrafas de malha de $5 \mathrm{~mm}$, em que foi padronizado o número de 15 repetições de cada método em cada fase de campo. Os espécimes foram colocados em sacos plásticos, etiquetados e fixados em formol $10 \%$. Posteriormente à fixação, essas amostras foram transferidas para solução de álcool 70\% e triadas no Laboratório de 

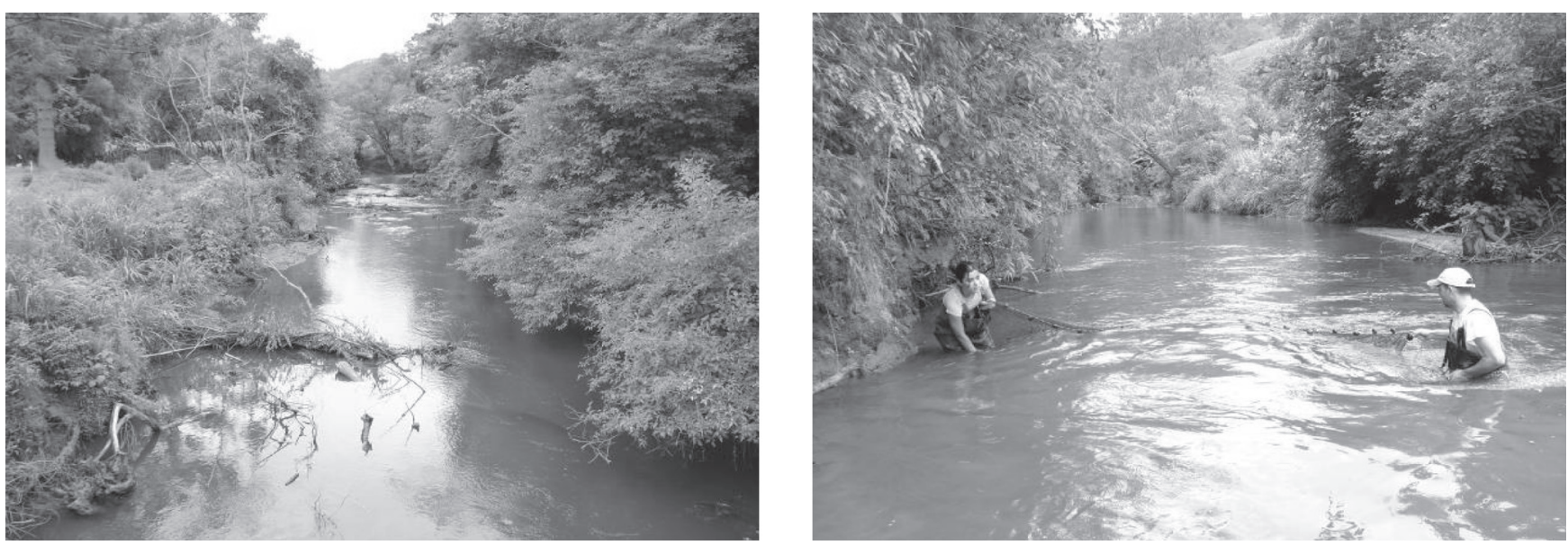

Figura 1 - Área de estudo no Rio das Almas, Ribeirão Grande, SP

Ictiologia do Museu de Historia Natural Capão da Imbuia (MHNCI). Em laboratório, os exemplares foram mensurados para a obtenção de comprimento padrão $(\mathrm{CP})$, pesados com auxílio de balança de precisão (0,001 gramas) e dissecados. Estômagos foram removidos e o conteúdo foi analisado sob microscópio estereoscópico. Os itens alimentares foram identificados até o menor nível taxonômico possível. Os itens alimentares foram agrupados em categorias taxonômicas e/ou ecológicas de acordo com sua origem: algas, ácaros aquáticos, estágios imaturos aquáticos de insetos (larvas e pupas), insetos aquáticos, crustáceos, oligoquetos, moluscos, nematoides e larvas de peixes foram considerados autóctones, enquanto insetos terrestres, aranhas, frutos e fragmentos de vegetais foram considerados alóctones.

Para a avaliação da abundância dos itens alimentares utilizou-se uma adaptação do método de pontos (10), em que a contribuição de cada item foi determinada pela proporção de quadrículas de $1 \mathrm{~mm}^{2}$ (pontos) ocupados pelo item em uma superfície plana quadriculada. Variações na composição e abundância na dieta entre os períodos amostrais e entre as classes de tamanho consideradas foram então avaliadas por meio de análises de escalonamento multidimensional não métrico (MDS) com sobreposição de cluster de ligação completa, análise de similaridade (ANOSIM) e análise de percentual de similaridade (SIMPER). As matrizes de similaridade com os valores de abundância dos itens alimentares foram geradas com o coeficiente de Bray-Curtis. As análises foram realizadas por meio do software Primer versão 6 (11).
Para a análise das variações ontogênicas da dieta, os indivíduos foram agrupados em quatro classes de comprimento padrão: classe 1 (menor que $3,5 \mathrm{~cm}$ ), classe 2 (entre 3,6 e 4,5 cm), classe 3 (entre 4,6 e 5,5 cm) e classe 4 (acima de 5,6 cm).

\section{Resultados}

Um total de 43 itens alimentares foi identificado nos estômagos de 262 exemplares capturados em junho de 2004, maio de 2006, novembro de 2006 e novembro de 2007. A análise do conteúdo estomacal revelou grande variedade de itens, os quais foram agrupados em 13 categorias (Tabela 1). As maiores riquezas de itens alimentares foram observadas para as categorias de estágios imaturos aquáticos de insetos (larvas e pupas) e insetos terrestres.

De acordo com a análise de similaridade (ANOSIM), os períodos de coleta não apresentaram variações significativas em relação à dieta $(\mathrm{R}=0,02$; $\mathrm{p}=0,26)$. Por outro lado, variações na abundância dos itens alimentares entre as classes de tamanho consideradas foram observadas $(\mathrm{R}=0,171 ; \mathrm{p}=$ 0,004). Para ambos os casos, a estatística R apresentou valores relativamente baixos, indicando sobreposição de dados. Observando o ordenamento produzido pela análise de escalonamento multidimensional não métrico (MDS) (Figura 2), a abundância dos itens alimentares entre as classes de tamanho consideradas não formaram de fato um marcado gradiente, $\mathrm{e}$ apenas os indivíduos menores que $3,5 \mathrm{~cm}$ (classe 1 ) formaram um agrupamento melhor definido. 
Esses resultados estão relacionados ao fato de que o consumo de insetos (terrestres e aquáticos) e vegetais foi registrado para todas as classes de tamanho consideradas, muito embora variações na abundância desses itens tenham sido registradas (Figura 3). De acordo com a análise de SIMPER, a maior dissimilaridade $(79,95 \%)$ na dieta foi observada entre os indivíduos menores que $3,5 \mathrm{~cm}$ (classe 1) e aqueles acima de $5,6 \mathrm{~cm}$ (classe 4 ), graças à representatividade dos itens vegetais, para os indivíduos maiores $(77,50 \%)$, e insetos aquáticos $(63,25 \%)$, para os indivíduos menores.

\section{Discussáo}

De acordo com os resultados obtidos, os insetos terrestres, estágios imaturos aquáticos de insetos e restos de vegetais superiores compõem a dieta do caracídeo $P$. argentea na região estudada, independentemente do período de coleta amostrado. Essas observações são concordantes com o trabalho de Ferreira et al. (7), que registrou como itens importantes insetos aquáticos, insetos terrestres e sementes. Esse hábito onívoro esteve provavelmente relacionado à captura de muitos exemplares em remansos cobertos por densa vegetação, pendente sobre o Rio das Almas, a qual forneceu recursos alóctones para a ictiofauna estudada.

Muito embora o regime estacional moderado da região permita a divisão em período chuvoso, com temperaturas mais altas, e outro parcialmente seco, com temperaturas médias mais baixas, a dieta não apresentou variações significativas entre os períodos amostrados. Por outro lado, alterações nos hábitos alimentares estiveram relacionadas com as classes de tamanho consideradas e os indivíduos maiores apresentaram tendência à herbivoria. Apesar dessa constatação, o consumo de insetos (terrestres e aquáticos) e vegetais foi registrado para todas as classes de tamanho consideradas e esse hábito alimentar onívoro parece ser estratégia predominante em pequenos cursos de água (12), podendo as diferenças nas abundâncias dos itens alimentares serem atribuídas a diferenças na disponibilidade de alimento.

A importância de recursos alóctones para as comunidades que compõem os ambientes de riachos localizados em áreas com mata ciliar preservada tem sido constantemente registrada (13-15). De acordo
Tabela 1 - Itens alimentares encontrados nos estômagos de $P$. argentea

(Continua)

\begin{tabular}{|c|c|}
\hline Origem & Item alimentar \\
\hline \multirow[t]{37}{*}{ Autóctones } & Arachnida \\
\hline & Acarinea \\
\hline & Estágios imaturos de insetos \\
\hline & Diptera (larva/pupa) - Chironomidae \\
\hline & Diptera (larva/pupa) - Simulidae \\
\hline & Diptera (larva/pupa) - Thaumaleidae \\
\hline & Diptera (larva/pupa) - Tabanidae \\
\hline & Diptera (larva/pupa) - Tipulidae \\
\hline & Diptera (larva) - Stratyomidae \\
\hline & Diptera (larva/pupa) - Ceratopogonidae \\
\hline & Ephemeroptera (larva) - Leptophebiidae \\
\hline & Ephemeroptera (larva) - Tricorythidae \\
\hline & Ephemeroptera (larva) - Baetidae \\
\hline & Ephemeroptera (larva) - Caenidae \\
\hline & Odonata (larva) - Coenagrionidae \\
\hline & Coleoptera (larva) - Elmidae \\
\hline & Trichoptera (larva) - Hydroptilidae \\
\hline & Trichoptera (larva) - Hydropsychidae \\
\hline & Trichoptera (larva) - Leptoceridae \\
\hline & Insetos aquáticos \\
\hline & Coleoptera (larva) - Mordellidae \\
\hline & Hemiptera - Belostomatidae \\
\hline & Hexapoda \\
\hline & Collembola \\
\hline & Crustacea \\
\hline & Isopoda \\
\hline & Aegla sp. \\
\hline & Annelida \\
\hline & Olygochaeta \\
\hline & Molusca \\
\hline & Bivalvia \\
\hline & Gastropoda \\
\hline & Nematoda \\
\hline & Vertebrata \\
\hline & Larva peixe \\
\hline & Escamas \\
\hline & Algas \\
\hline
\end{tabular}


Tabela 1 - Itens alimentares encontrados nos estômagos de $P$. argentea

(Conclusão)

\begin{tabular}{ll}
\hline Origem & Item alimentar \\
\hline Alóctones & Frutos e fragmentos vegetais \\
& Insetos terrestres \\
& Coleoptera - Elmidae \\
& Coleoptera - Carabidae \\
& Coleoptera - Herotylidae \\
& Coleoptera \\
& Hymenoptera - Formicidae \\
& Hymenoptera - Vespidae \\
& Hymenoptera - Vespidae \\
& Hemiptera - Belostomatidae \\
& Hemiptera - Psyllidae \\
& Thysanoptera \\
& Diptera - Psychodidae \\
& Diptera \\
& Arachnida \\
Aranea
\end{tabular}

Fonte: Dados da pesquisa.

com Castro (3), como os riachos possuem produtividade primária relativamente baixa e os processos são predominantemente heterotróficos, a ictiofauna desses ambientes depende da importação da matéria alóctone para a sua subsistência. Dessa forma, nesses sistemas as matas ciliares exercem grande importância no que se refere à fonte de alimentos, habitats e refúgios, que são fatores importantes para a manutenção da diversidade da fauna aquática $(8$, $9,16,17)$.

Os resultados deste estudo apontam para a importância da conservação dos riachos de cabeceiras na Bacia do Rio Paraná, visto que os fenômenos que ocorrem nesse tipo de ecossistema aquático determinam alterações no ciclo biológico de diversas espécies que utilizam esses ambientes similares. Tendo isso em vista, a preservação de remanescentes na área de estudo, mesmo que isolados, tem papel importante na manutenção da diversidade faunística local, proporcionando uma área que compreende uma série de espécies dependentes, em maior ou menor grau, de ambientes florestais.

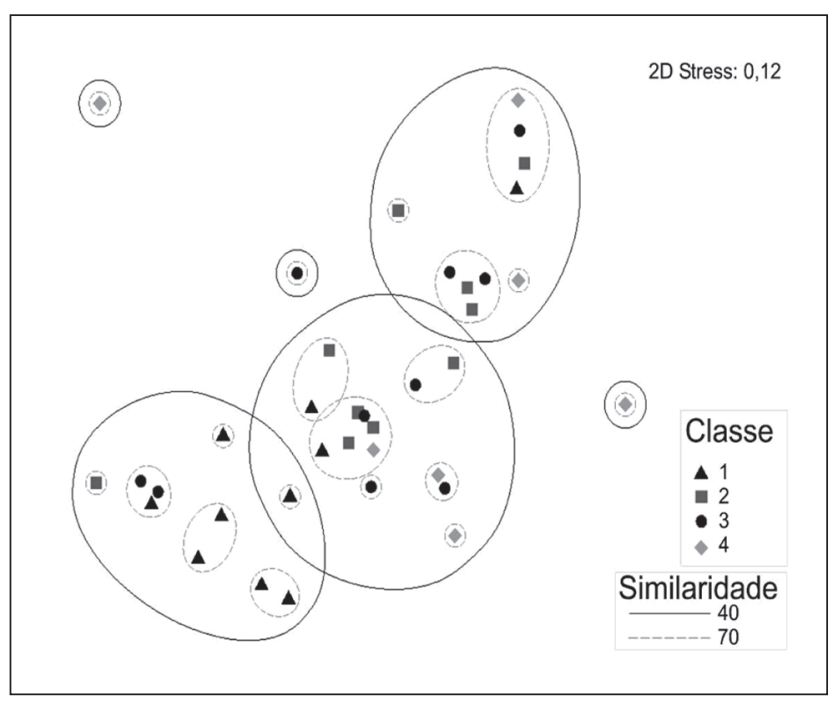

Figura 2 - Representação gráfica bidimensional de distribuição dos itens alimentares por meio da análise de escalonamento multidimensional não métrico (MDS) com sobreposição de cluster de ligação completa. Os traços representam o grau de similaridade obtido por meio da análise de cluster. Classe $1(<3,5 \mathrm{~cm})$, classe 2 (entre 3,6 e 4,5 cm), classe 3 (entre 4,6 e 5,5 cm) e classe $4(>5,6 \mathrm{~cm})$

Fonte: Dados da pesquisa.

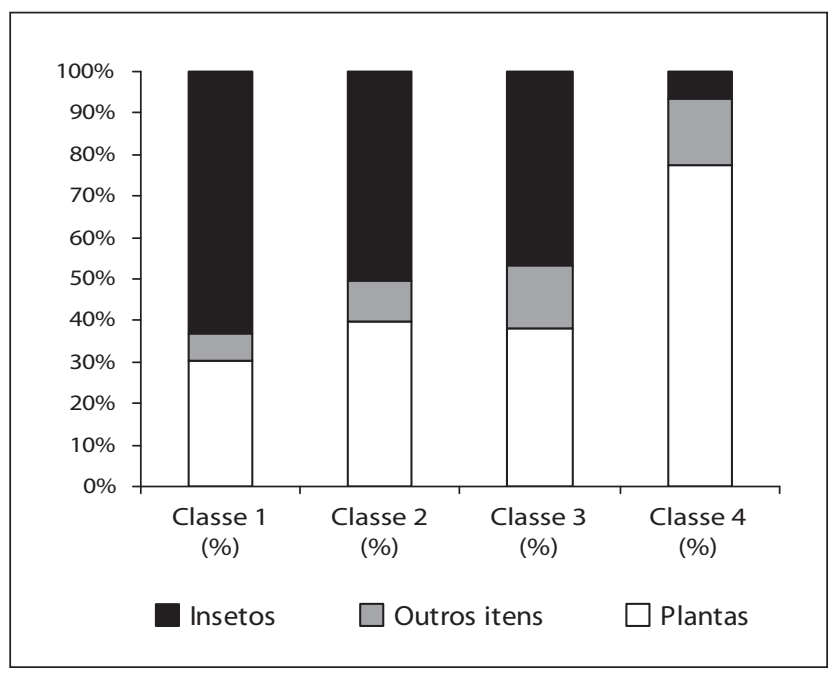

Figura 3 - Contribuição percentual dos insetos, plantas e outras categorias de itens alimentares para a dieta de $P$. argentea, nas quatro classes de tamanho analisadas. Classe $1(<3,5 \mathrm{~cm})$, classe 2 (entre 3,6 e 4,5 cm), classe 3 (entre 4,6 e 5,5 cm) e classe $4(>5,6 \mathrm{~cm})$

Fonte: Dados da pesquisa. 


\section{Referências}

1. Graça WJ, Pavanelli CS. Peixes da planície de inundação do Alto Rio Paraná e áreas adjacentes. Maringá: EDUEM; 2007.

2. Castro RMC, Menezes NA. Estudo diagnóstico da diversidade de peixes do Estado de São Paulo. In: Castro RMC, Joly CA, Bicudo CEM, editores. Biodiversidade do Estado de São Paulo, Brasil: síntese do conhecimento ao final do século XX, vol. 6 vertebrados. São Paulo: FAPESP; 1998.

3. Castro RMC. Evolução da ictiofauna de riachos sulamericanos: padrões gerais e possíveis processos causais. In: Caramaschi EP, Mazzoni R, Perez-Neto PR, editores. Ecologia de peixes de riachos. Rio de Janeiro: PPGE-UFRJ; 1999.

4. Lowe-McConnell RH. Estudos ecológicos de comunidades de peixes tropicais. São Paulo: EDUSP; 1999.

5. Sabino J, Castro RMC. Alimentação, período de atividade e distribuição espacial dos peixes de um riacho da Floresta Atlântica (Sudeste do Brasil). Rev Bras Biol. 1990;50(1):23-36.

6. Araújo-Lima CARM, Agostinho AA, Fabré N. Trophic aspects of fish communities in Brazilian rivers and reservoirs. In: Tundisi JG, Bicudo CEM, Matsumura-Tundisi T, editores. Limnology in Brazil. Rio de Janeiro: ABC/SBL; 1995.

7. Ferreira A, Hahn NS, Delariva RL. Ecologia alimentar de Piabina argentea (Teleostei, Tetragonopterinae) nas fases de pré e pós-represamento do rio Corumbá, GO. Acta Limnologica Brasiliensia. 2002;14(1): 43-52.

8. Crowder LB, Cooper WE. Habitat structural complexity and the interaction between bluegills and their prey. Ecology. 1982;63(6):1802-13.

9. Gilinsky E. The role of fish predation and spatial heterogeneity in determining benthic community structure. Ecology. 1984;65(2):455-68.
10. Hynes HBN. The food of fresh-water sticklebacks (Gasterosteus aculeatus and Pygosteus pungitius), with a review of methods used in studies of the food of fishes. J Anim Ecology. 1950;19(1):36-51.

11. Clarke KR, Gorley RN. PRIMER v6: user manual/ tutorial. Plymouth: PRIMER-E; 2006.

12. Pringle $\mathrm{CM}$, Hamazaki $\mathrm{T}$. The role of omnivory in a neotropical stream: separating diurnal and nocturnal effects. Ecology. 1988;79:269-80.

13. Casatti L. Alimentação dos peixes em um riacho do Parque Estadual Morro do Diabo, bacia do alto rio Paraná, sudeste do Brasil. Biota Neotropica. 2002;2(2):1-14.

14. Mazzoni R, Rezende CF. Seasonal diet shift in a Tetragonopterinae (Osteichthyes, Characidae) from Ubatiba river, RJ, Brazil. Brazilian J Biology. 2003;63(1):69-74.

15. Rezende CF, Mazzoni R. Disponibilidade e uso de recursos alóctones por Bryconamericus microcephalus (Miranda-Ribeiro) (Actinopterygii, Characidae), no córrego Andorinha, Ilha Grande, Rio de Janeiro, Brasil. Revi Bras Zool. 2006;23(1):218-22.

16. Gotceitas V, Colgan P. Predator foraging success and habitat complexity: quantitative test of the threshold hypothesis. Oecologia. 1989;80:158-66.

17. Vono V, Barbosa FAR. Habitats and littoral zone fish community structure of two natural lakes in Southeast Brazil. Environmental Biology of Fishes. 2001;61(4):371-79.

Recebido: 22/03/2009

Received: 03/22/2009

Aprovado: 14/10/2009

Approved: 10/14/2009 